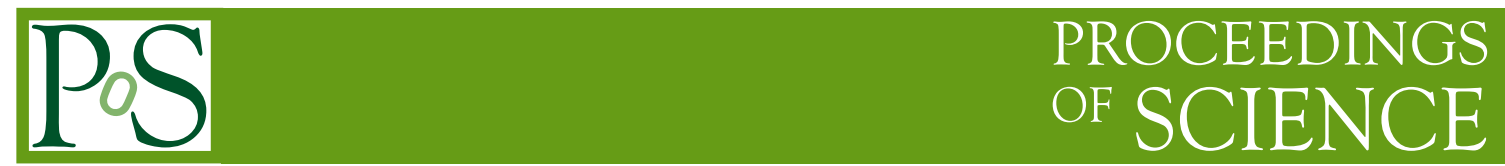

\title{
Deriving confinement via RG decimations
}

\author{
E.T. Tomboulis* \\ Univ of California, Los Angeles \\ E-mail: tombouliephysics.ucla.edu
}

We present the general framework and building blocks of a recent derivation of the fact that the $S U$ (2) LGT is in a confining phase for all values of the coupling $0<\beta<\infty$, for space-time dimension $d \leq 4$. The method employs approximate but explicitly computable RG decimations that are shown to constrain the exact partition function and order parameters from above and below, and flow from the weak to the strong coupling regime without encountering a fixed point.

The XXV International Symposium on Lattice Field Theory

July 30 - August 42007

Regensburg, Germany

${ }^{*}$ Speaker. 


\section{Introduction}

4-dim. $S U(N)$ gauge theory at $T=0$ is known to be in a single phase for all values of the gauge coupling, $0<\beta<\infty$. This fact has proved hard to derive directly. This is not surprising since one is faced with a multi-scale problem involving passage from a short distance perturbative ordered regime to a long distance non-perturbative confining disordered regime. The natural framework here is RG block-spinning bridging these disparate regimes. Ideally, one would like to construct an exact block-spinning scheme converging to the 'perfect action' governing the Wilsonian renormalized trajectory. Then one would be able to compute any observable at different scales. Despite some valiant efforts, however, this has proven technically too complicated to carry out so far.

Progress can be made, nonetheless, by adopting a more modest but still sufficiently general framework. This approach employs approximate, rather than exact, but easily explicitly computable decimation procedures that can provide bounds on judicially chosen quantities. Such quantities are partition functions (free energies) and their ratios (free energy differences). The basic idea is to consider only such quantities - they can serve as order parameters - and not attempt to construct a general RG blocking action suitable, in principle, for computing any observable. One then uses the bounds to fix the behavior of corresponding exact quantities by interpolating between the bounds. In this fashion one can derive statements concerning the exact theory, and in particular the presence of the confining phase. A detailed account has recently appeared in [1]. Here we present an outline of the basic steps involved in this development.

\section{Partition function}

We employ standard lattice gauge theory (LGT) notations, $U$ denoting generic group elements, $U_{b}$ bond variables, $U_{p}=\prod_{b \in \partial p} U_{b}$, etc. Starting with a plaquette action, for example the Wilson action

$$
A_{p}\left(U_{p}\right)=\frac{\beta}{2} \chi_{1 / 2}\left(U_{p}\right)
$$

the character expansion of the exponential of the action is:

$$
e^{A_{p}(U)}=\sum_{j} d_{j} F_{j}(\beta) \chi_{j}(U)
$$

For $S U(2)$, the only case considered explicitly here, $j=0, \frac{1}{2}, 1, \frac{3}{2}, \ldots$, and $d_{j}=(2 j+1)$. In terms of normalized coefficients $c_{j}(\beta)=F_{j}(\beta) / F_{0}(\beta)$ :

$$
e^{A_{p}(U)}=F_{0}\left[1+\sum_{j \neq 0} d_{j} c_{j}(\beta) \chi_{j}(U)\right] .
$$

The Partition Function (PF) on lattice $\Lambda$ of spacing $a$ is then defined by

$$
Z_{\Lambda}(\beta)=\int d U_{\Lambda} \prod_{p}\left[1+\sum_{j \neq 0} d_{j} c_{j}(\beta) \chi_{j}(U)\right] \equiv Z_{\Lambda}\left(\left\{c_{j}(\beta)\right\}\right)
$$

For a reflection positive action:

$$
F_{j} \geq 0 \quad \text { hence } \quad 1 \geq c_{j} \geq 0 \quad \text { all } \quad j .
$$


We now introduce decimations whereby the lattice spacing is changed by a scale factor $b$. We employ decimation operations of the 'bond moving type' [2], [3], which preserve the form (2.4]. Such a decimation operation can be summarized as a set of decimation rules for each successive step:

$$
\begin{aligned}
& a \rightarrow b a \rightarrow b^{2} a \rightarrow \cdots \rightarrow b^{n} a \\
& \Lambda \rightarrow \Lambda^{(1)} \rightarrow \Lambda^{(2)} \rightarrow \cdots \rightarrow \Lambda^{(n)}
\end{aligned}
$$

from lattice $\Lambda^{(m)}$ of spacing $b^{m} a$ to lattice $\Lambda^{(m+1)}$ of spacing $b^{m+1} a$, with $\Lambda^{(0)}=\Lambda$.

The rules provide an explicit expression for the computation of the Fourier coefficients at the $m+1$-th step given those of the $m$-th step:

$$
\begin{aligned}
F_{0}(m) & =F_{0}\left(\zeta, r, b,\left\{c_{i}(m-1)\right\}\right) \\
c_{j}(m) & =c_{j}\left(\zeta, r, b,\left\{c_{i}(m-1)\right\}\right) .
\end{aligned}
$$

The explicit form of (2.6) - (2.7) need not be given here; we only note that they involve parameters $\zeta, r$ which control the amount by which the interactions of the plaquettes remaining after a decimation step are 'renormalized' to compensate for the ones that were removed.

Correspondingly, the partition function undergoes the transformation

$$
Z_{\Lambda^{(m-1)}}\left(\left\{c_{j}(m-1)\right\}\right) \rightarrow F_{0}(m)^{\left|\Lambda^{(m)}\right|} Z_{\Lambda^{(m)}}\left(\left\{c_{j}(m)\right\}\right) .
$$

There is a bulk free energy contribution from the blocking $b^{m-1} a \rightarrow b^{m} a$. The resulting effective action in $Z_{\Lambda^{(m)}}\left(\left\{c_{j}(m)\right\}\right)$ on the resulting lattice $\Lambda^{(m)}$ retains the original one-plaquette form. It will, however, contain, in general, all group representations even after just one decimation step starting from an action containing one or a finite number of representations:

$$
\exp \left[A_{p}\left(U_{p}, m\right)\right]=\left[1+\sum_{j \neq 0} d_{j} c_{j}(m) \chi_{j}\left(U_{p}\right)\right]
$$

with

$$
A_{p}(U, m)=\sum_{j} \beta_{j}(m) \chi_{j}(U) .
$$

Also, both positive and negative effective couplings $\beta_{j}(m)$ will in general occur. But in (2.9) all $c_{j}(m) \geq 0$ if the parameter $\zeta=$ integer. This implies that reflection positivity of the measure is preserved after each decimation step.

Upper and lower bounds In going from the $(m-1)$-th step to the $m$-th decimation step consider the following choices of decimation parameters in (2.6) - (2.7):

- (I) $\zeta=b^{d-2}, \quad r=1-\varepsilon, \quad 0 \leq \varepsilon<1$; denote the resulting $m$-th step coefficients by $F_{0}^{U}(m)$ and $c_{j}^{U}(m)$. This is essentially (for $r=1$ ) the choice made in [2] - [3].

- (II) $\zeta=1, \quad r=1$; denote the resulting coefficients by $F_{0}^{L}(m)$ and $c_{j}^{L}(m)$. 
Then one has the following basic inequalities relating the PF's before and after the decimation:

$$
F_{0}^{L}(m)^{\left|\Lambda^{(m)}\right|} Z_{\Lambda^{(m)}}\left(\left\{c_{j}^{L}(m)\right\}\right)<Z_{\Lambda^{(m-1)}}<F_{0}^{U}(m)^{\left|\Lambda^{(m)}\right|} Z_{\Lambda^{(m)}}\left(\left\{c_{j}^{U}(m)\right\}\right) .
$$

A variety of similar bounds can be employed (i.e. somewhat different definitions of $F_{0}^{U, L}(m)$ and $\left.c_{j}^{U, L}(m)\right)$ in (2.11) (cf. [1]). Such technical details are not important for the general development below.

Interpolation Introducing a parameter $\alpha, 0 \leq \alpha \leq 1$, define interpolating coefficients $\tilde{c}_{j}(m, \alpha)$ and $\tilde{F}_{0}(m, \alpha)$ such that

$$
\tilde{c}_{j}(m, \alpha)=\left\{\begin{array}{l}
c_{j}^{U}(m): \alpha=1 \\
c_{j}^{L}(m): \alpha=0
\end{array},\right.
$$

and similarly

$$
\tilde{F}_{0}(m, \alpha)=\left\{\begin{array}{l}
F_{0}^{U}(m): \alpha=1 \\
F_{0}^{L}(m): \alpha=0
\end{array}\right.
$$

There is clearly an infinity of such smooth interpolations that can be defined. But there is nothing unique about any one such interpolation. It is expedient then to consider more generally a family of smooth interpolations parametrized by a parameter $t$ in some interval $\left(t_{1}, t_{2}\right)$.

Then the upper-lower bounds statement (2.11) implies that, for each value of $t$ picking an interpolation family member, there exist some value of the interpolating parameter $\alpha=\alpha_{\Lambda}^{(m)}(t)$, where

such that

$$
0<\alpha_{\Lambda}^{(m)}(t)<1
$$

$$
\tilde{F}_{0}(m, \alpha, t)^{\left|\Lambda^{(m)}\right|} Z_{\Lambda^{(m)}}\left(\left\{\tilde{c}_{j}(m, \alpha)\right\}\right)=Z_{\Lambda^{(m-1)}} .
$$

Note that, by construction, there is parametrization invariance under shift in $t$ in the 1.h.s. of (2.14); in other words, $\alpha=\alpha_{\Lambda}^{(m)}(t)$ is the level surface fixed by (2.14). Furthermore, one can show that

$$
\alpha_{\Lambda}^{(m)}(t)=\alpha^{(m)}(t)+\delta \alpha_{\Lambda}^{(m)}(t), \quad \text { with } \quad \delta \alpha_{\Lambda}^{(m)}(t) \rightarrow 0, \quad|\Lambda| \rightarrow \infty
$$

and lattice-volume independent $\alpha^{(m)}(t)$.

So, iterating this procedure starting from the original lattice, one gets an exact integral representation of the PF on successively decimated lattices:

$$
\begin{aligned}
Z_{\Lambda}(\beta) & =Z_{\Lambda}\left(\left\{c_{j}(\beta)\right\}\right) \\
& =\tilde{F}_{0}\left(1, \alpha_{\Lambda}^{(1)}\left(t_{1}\right), t_{1}\right)^{\left|\Lambda^{(1)}\right|} Z_{\Lambda^{(1)}}\left(\left\{\tilde{c}_{j}\left(1, \alpha_{\Lambda}^{(1)}\left(t_{1}\right)\right)\right\}\right) \\
& =\cdots \\
& =\left[\prod_{m=1}^{n} \tilde{F}_{0}\left(m, \alpha_{\Lambda}^{(m)}\left(t_{m}\right), t_{m}\right)^{|\Lambda| / b^{d m}}\right] Z_{\Lambda^{(n)}}\left(\left\{\tilde{c}_{j}\left(n, \alpha_{\Lambda}^{(n)}\left(t_{n}\right)\right)\right\}\right)
\end{aligned}
$$

The representation is in terms of the accumulated bulk free energy contributions from the successive blockings from scale $a$ to scale $b^{n} a$, and the resulting effective action and corresponding PF on $\Lambda^{(n)}$. Note that all coefficients occurring in this representation are constrained by the bounding coefficients that are computable by the decimation rules (2.6) - (2.7).

There are many potential uses for exact representations such as (2.16) and its derivatives. In the following we use it to examine the confining properties of the theory. 


\section{3. 'Twisted' partition function}

Let $\mathscr{V}$ denote a coclosed set of plaquettes winding around the periodic lattice in the $d-2$ directions normal to, say, the [12]-plane. Let $Z_{\Lambda}^{(-)}$denote the partition function with action on every plaquette in $\mathscr{V}$ shifted by a non-trivial element $\tau$ ('twist') of the group center. Thus, for $S U(2), \tau=-1 \in Z(2)$. The twist represents the introduction of external $\pi_{1}(S U(2) / Z(2))=Z(2)$ vortex flux in $\Lambda$. For $Z_{\Lambda}^{(-)}$reflection positivity holds only in planes perpendicular to the directions in which $\mathscr{V}$ winds around the lattice. To have RP in all planes one may simply replace $Z_{\Lambda}^{(-)}$by $Z_{\Lambda}^{+} \equiv \frac{1}{2}\left(Z_{\Lambda}+Z_{\Lambda}^{(-)}\right)$

The above development can then be carried through also for $Z_{\Lambda}^{+}$applying the same decimations (2.6) - (2.7), obtaining the analog of (2.11) giving upper and lower bounds in terms of $F_{0}^{U, L}(\mathrm{~m})$ and $c_{j}^{U, L}(m)$, and interpolating between them. One thus obtains the corresponding integral representation on successively decimated lattices:

$$
\begin{aligned}
Z_{\Lambda}^{+}= & {\left[\prod_{m=1}^{n} \tilde{F}_{0}\left(m, \alpha_{\Lambda}^{+(m)}\left(t_{m}\right), t_{m}\right)^{|\Lambda| / b^{d m}}\right] } \\
& \cdot \frac{1}{2}\left[Z_{\Lambda^{(n)}}\left(\left\{\tilde{c}_{j}\left(n, \alpha_{\Lambda}^{(+)}\left(t_{n}\right)\right)\right\}\right)+Z_{\Lambda^{(n)}}^{(-)}\left(\left\{\tilde{c}_{j}\left(n, \alpha_{\Lambda}^{+(n)}\left(t_{n}\right)\right)\right\}\right)\right] .
\end{aligned}
$$

Again, one can show that

$$
\alpha_{\Lambda}^{+(m)}(t)=\alpha^{(m)}(t)+\delta \alpha_{\Lambda}^{+(m)}(t), \quad \text { with } \quad \delta \alpha_{\Lambda}^{+(m)}(t) \rightarrow 0, \quad|\Lambda| \rightarrow \infty .
$$

As can be seen from (3.1), the external flux presence does not affect the bulk free-energy contributions that resulted from successive blockings. Also, it should be noted that, as indicated by the notation, the values $\alpha_{\Lambda}^{+(m)}(t)$ fixed at each successive step $m=1, \ldots, n$ in this representation are a priori distinct from the values $\alpha_{\Lambda}^{(m)}(t)$ in the representation 2.16 for $Z_{\Lambda}(\beta)$. This is because they are fixed by an independent procedure involving a distinct quantity. It is easily seen, however, that any discrepancies between $\alpha_{\Lambda}^{+(m)}(t)$ and $\alpha_{\Lambda}^{(m)}(t)$ can only occur in the lattice-dependent parts $\delta \alpha_{\Lambda}^{+(m)}(t), \delta \alpha_{\Lambda}^{(m)}(t)$. This assumes that the same family of interpolations is used in (2.16) and (3.1). In general, however, one may of course make different choices of interpolation in the two cases.

\section{Order parameters - Vortex free energy}

The vortex free-energy is defined as:

$$
\exp \left(-F_{\Lambda}(\beta)\right)=\frac{Z_{\Lambda}^{(-)}}{Z_{\Lambda}}
$$

Physically, it represents the difference in free energies between the vacuum in the presence and in the absence of an externally introduced $\pi_{1}(S U(2) / Z(2)$ ) vortex. As it is well-known, (4.1) serves as an order parameter characterizing the phases of gauge theory [ $₫$. It is known, in particular, that confining behavior for (4.1) implies confining behavior (area law) of the Wilson loop [5]. 
One may now represent this ratio on successively decimated lattices by inserting our representations (2.16), (3.1) in the numerator and denominator in

$$
\left(1+\frac{Z_{\Lambda}^{(-)}}{Z_{\Lambda}}\right)=\frac{Z_{\Lambda}+Z_{\Lambda}^{(-)}}{Z_{\Lambda}} .
$$

Comparing these representations one sees that any discrepancies between $\alpha_{\Lambda}^{+(m)}(t)$ and $\alpha_{\Lambda}^{(m)}(t)$, even if they vanish in the large volume limit (cf. (2.15), (3.2)), can leave residual non-vanishing effects from bulk free energy contributions not completely canceling between numerator and denominator in (4.2). One may, however, utilize the independent invariance under parametrization shifts in numerator and denominator to arrange for complete cancellation of the bulk free energy pieces between numerator and denominator generated at each step.

Carrying out $n$ decimation steps with $n$ sufficiently large one ends up with

$$
\frac{Z_{\Lambda}^{(-)}}{Z_{\Lambda}}=\frac{Z_{\Lambda^{(n)}}^{(-)}\left(\left\{\tilde{c}_{j}\left(n, \alpha_{\Lambda}^{*(n)}\right)\right\}\right)}{Z_{\Lambda^{(n)}}\left(\left\{\tilde{c}_{j}\left(n, \alpha_{\Lambda}^{*(n)}\right)\right\}\right)} .
$$

Here $\alpha_{\Lambda}^{*(n)}$ denotes $\alpha_{\Lambda}^{(n)}(t)$ at a particular $t=t^{*}$ such that $\alpha_{\Lambda}^{(n)}\left(t^{*}\right)=\alpha_{\Lambda}^{+(n)}\left(t^{*}\right)$ after a final interpolation parametrization shift in the numerator versus the denominator.

Now, by construction, the coefficients in terms of which the PF's in (4.3) are computed are bounded by the upper bound coefficients:

$$
\tilde{c}_{j}\left(n, \alpha_{\Lambda}^{*(n)}\right) \leq c_{j}^{U}(n) .
$$

Recall that the upper bound coefficients are explicitly computable in terms of the decimation rules, (2.6), (2.7). For the type of potential moving decimations used here it is a known fact that, for $S U(2)$, one finds

$$
c_{j}^{U}(n) \rightarrow 0 \quad \text { for } \quad n \rightarrow \infty,
$$

provided the space-time dimensionality $d \leq 4$. (4.4), (4.5) imply then that, by taking the number of decimations $n$ large enough, one can compute the resulting expression (4.3) within the convergent strong coupling expansion.

In this manner one arrives at the following:

- The vortex free energy parameter (4.1) exhibits confining behavior for any initial $\beta$ and $d \leq 4$. This follows from the fact that (4.5) holds for any initial $\beta$.

- Fixing the resulting string tension $\kappa(\beta, n)$ implies a relation between $n$ and $\beta=2 / g^{2}$.

- Now zero coupling $g=0$ is a fixed point of the decimations. This implies that to reach any fixed value of the string tension (some given value of $c_{j}^{U}(n)$ 's) requires

$$
\beta \rightarrow \infty \Longleftrightarrow n \rightarrow \infty \text {. }
$$

In other words one necessarily has

$$
g(a) \rightarrow 0 \quad \text { for } \quad a \rightarrow 0
$$

as an essentially qualitative feature of the decimation flow. 


\section{Summary}

A framework was developed within LGT that utilizes approximate but explicitly computable RG decimations which provide bounds constraining the exact theory. By a process of interpolation between such lower and upper bounds statements concerning the behavior of the exact theory can be obtained. This has many potential applications.

This framework was applied to the $S U(2)$ gauge theory. Exact integral representations of the PF with or without external flux on successively coarser lattices were obtained. There are many potential uses for such representations. They were used here to examine the so-called vortex freeenergy order parameter which can characterize the phases of the theory. Confinement at $T=0$ emerges for any initial coupling in four space-time dimensions once the approximate bounding decimations possess this property.

Extension to $S U(3)$ should be straightforward - the approximate decimations exhibit the same qualitative flow as in the $S U(2)$ case. In the above we were able to extract statements about the exact theory without actually knowing the actual numerical values of the interpolating parameters in the exact PF representations. Developing methods for numerical approximation of these values would be very useful, for example in conjunction with MCRG (Monte Carlo RG) techniques.

\section{Acknowledgments}

This work was partially supported by NSF-PHY-0555693.

\section{References}

[1] E.T. Tomboulis, arXiv:0707.2179 [hep-th].

[2] A.A. Migdal, ZhETF (USSR) 69, 810; 1457 (1975) [JETP (Sov. Phys.) 42, 413; 743 (1976)].

[3] L.P. Kadanoff, Ann. of Phys. 100, 359 (1976).

[4] G. 't Hooft, Nucl. Phys. B138, 1 (1978); ibid. B153, 141 (1979).

[5] T. Kovács and E.T. Tomboulis, Phys. Rev. D65, 074501 (2002) [arXiv:hep-lat/0108017]. 